\title{
Understanding Complex Microstructures With High-Resolution Microanalysis in the Transmission Electron Microscope.
}

\author{
G.A. Botton*, J. A. Gianetto**, C. V. Hyatt" and M.W. Phaneuf ${ }^{\S}$. \\ *Dept of Materials Science and Engineering, McMaster University, Hamilton, ON, L8S 4M1, Canada; ** Materials \\ Technology Laboratory-CANMET, Natural Resources Canada, Ottawa, ON, Canada; "Defense Research and \\ Development Canada, DREA, Halifax, NS, Canada; ${ }^{\S}$ Fibics Incorporated, 556 Booth, Ottawa, ON, K1A 0G1, Canada.
}

The development of intermediate voltage transmission electron microscopes equipped with fieldemission sources, have made analytical transmission electron microscopy (TEM) a widely used tool in the characterization of fine microstructures. In the general area of metallography, however, TEM is often considered as a tool of last resort to characterize complex materials because of the difficulty associated with sample preparation and limited sampling of the microstructures. With access to new sample preparation tools such as the focused ion beam (FIB), metallographic analysis of samples by TEM is likely to become a routine tool to elucidate not only complex materials but also microstructural and chemical nature of materials subjected to environmental degradation. Specific regions in welded microstructures or areas showing preferential corrosion can be selected and analyzed at high spatial resolution. In this short abstract some examples illustrate the possibilities of identifying features in such complex microstructures that would not have been possible without making use of the high spatial resolution analysis available in analytical TEM. Previous work ${ }^{(1)}$ has shown the potential of TEM and FIB to identify the response of various phases to electrochemical noise tests in saline water.

To further demonstrate this approach we discuss an example of analysis taken from on-going work related to the study of laser clad nickel aluminum bronzes (NAB) that were subjected to marine corrosion testing. In this work, laser clad coupons were subjected to alternately flowing and stagnant long-term seawater immersion testing for 1 to 4 years. To obtain a more fundamental understanding of the corrosion behaviour of the laser clad welds the thin corrosion product and the first few microns from the sample surface were analyzed ${ }^{(2)}$. In the cross-sections of the sample, the corrosion product is clearly visible and fully preserved (Fig. 1). Measurements of the energy loss fine structures at the oxygen K edge reveal that the corrosion product contains hydroxides fully preserved in spite of the sample preparation used to obtain the thin sections (Fig. 2). Another area of interest along the laserclad surface included a region where less obvious attack had occurred in the proximity of a grain boundary (Fig. 3a). This area was investigated to assess whether preferential corrosion occurs where boundaries intersect the surface of the sample. Although such effect was not observed, the high resolution energy dispersive x-ray spectrometry (EDXS) elemental mapping (Fig. 3b, c, d) demonstrated very clearly the depletion of Fe containing $\kappa_{\text {iv }}$ precipitates $(20-50 \mathrm{~nm}$ in size) in proximity of the grain boundaries and near the larger precipitates. Such effect was not detected in the conventional TEM or in the annular dark-field micrographs. Al was detected at the sample surface.

This brief example shows the potential of the combination of analytical TEM with FIB to study complex microstructures and reveal features not visible by other analytical means typically used in conventional metallography techniques. Further examples related to corrosion studies of NAB and Ni based alloys will also be discussed.

(1) R.D. Klassen, et al. Can. Metal. Quart. 41, 1, p 121-132, 2002.

(2) J.A. Gianetto et al. Special Report DREA SR 2001-094, p.67-82, October 2001. 

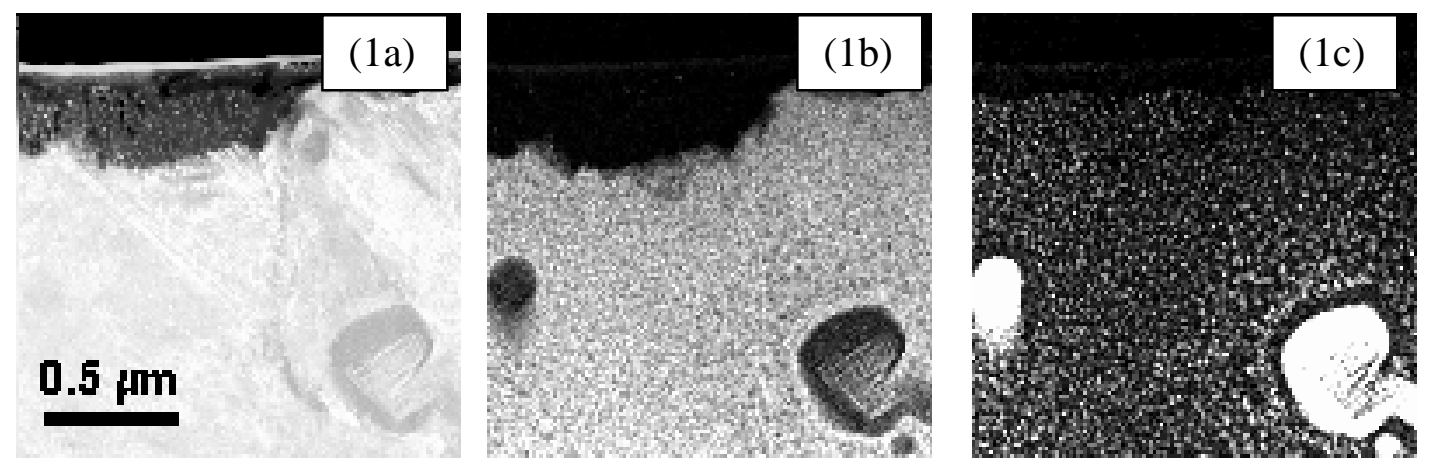

$\mathrm{O} K$ edge in layer $\mathrm{w}$ particles

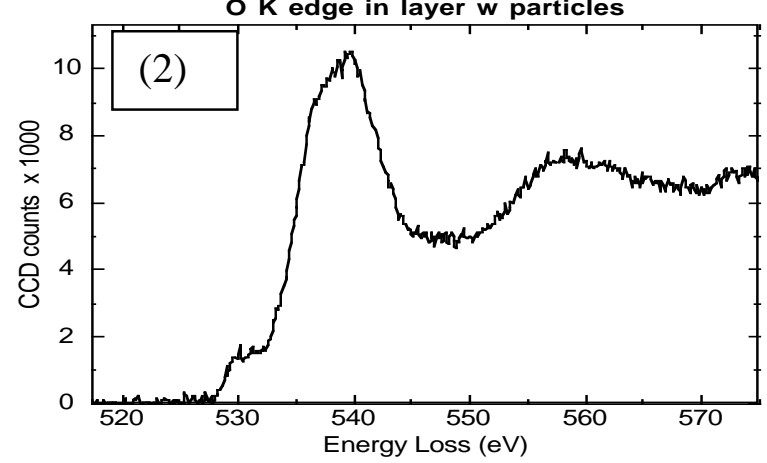

Figure 1. a) Dark-field image of an area with enhanced corrosion product in a NiAl bronze submitted to sea-water corrosion. EDXS elemental maps of $\mathrm{Cu}$ map (b) and Fe map (c).

Figure 2. Near-edge fine structure of the $\mathrm{O} \mathrm{K}$ edge in the area with enhanced corrosion. The broad peak and the shoulder at $530 \mathrm{eV}$ suggest the presence of hydroxides

Figure 3.

Microstructure and high-resolution elemental maps of the uniform layer formed on the surface of a NiAl bronze. a) Darkfield image, b) $\mathrm{Cu}$ elemental map, c) Fe elemental map, and d) Cr elemental map showing depletion of $\kappa_{\mathrm{IV}}$ precipitates in proximity of large precipitates and near a grain boundary in the centre of the field of view.
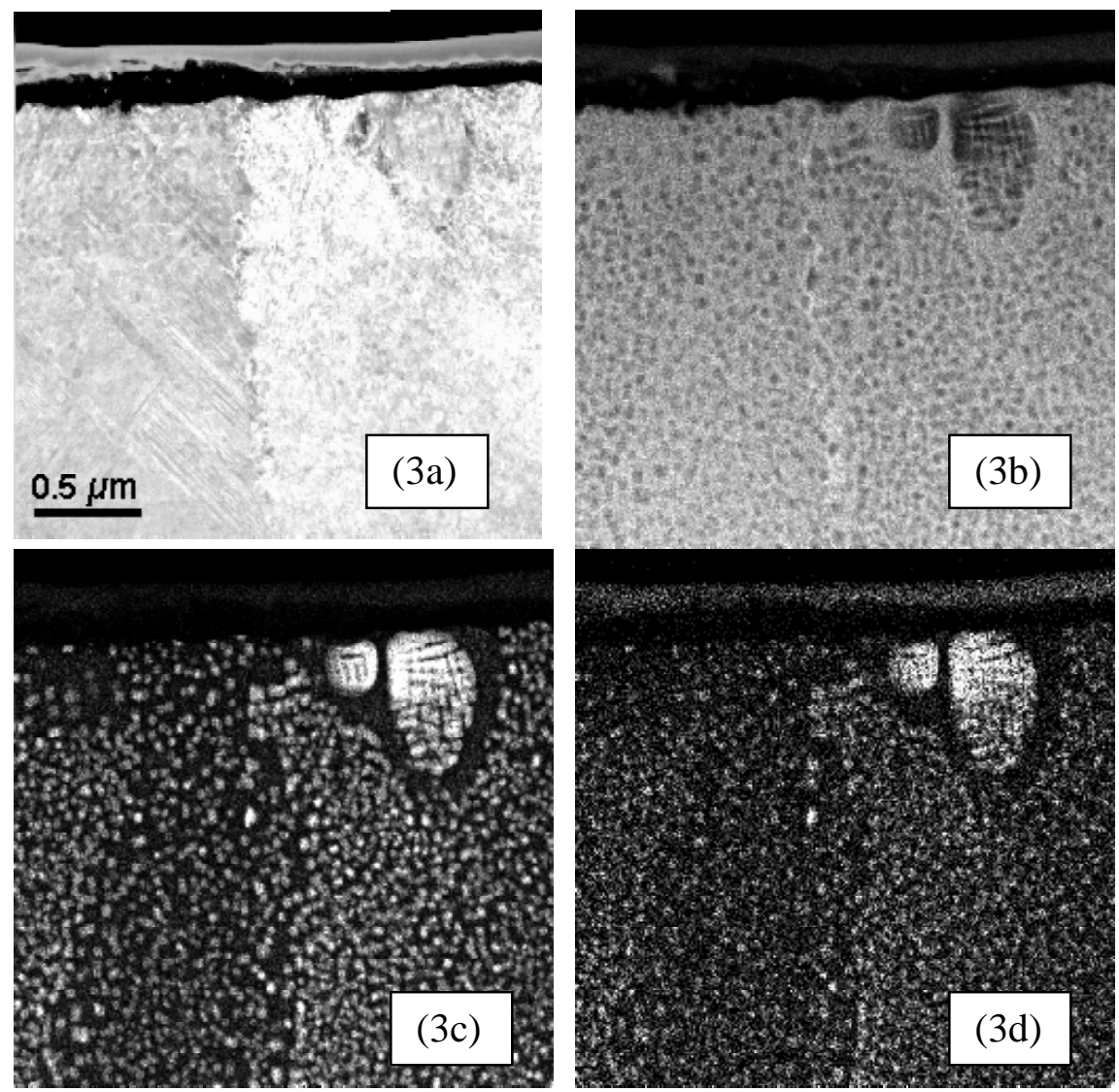